\title{
A NEW INTERACTIVE RAILWAY VIRTUAL SIMULATOR FOR TESTING PREVENTIVE SAFETY
}

\author{
STEFANO PAPA, ANTONIO LANZOTTI, GIUSEPPE DI GIRONIMO \& ALESSIO BALSAMO \\ Fraunhofer JL IDEAS, Department of Industrial Engineering, University of Naples Federico II, Italy
}

\begin{abstract}
The objective of the work is to describe the design and the realization of a virtual simulator of a metropolitan railway cockpit, aimed at improving the perception of safety by means of tests made by users in Virtual Reality, analysed through statistical methodologies. The user lives the experience of a driver in an immersive and interactive Augmented Reality session, interacting with the train dashboard and all its control and signalling devices. In particular, the user is proposed to test different dashboards, different configurations of the controls and different signalling and safety devices in order to compare different concept and select the optimum in terms of perception of dangerous situation, reaction to an event and cognitive response in different situations of the rail vehicle driving. The simulator consists of a simulacrum integrating different technologies, physically composed of a dashboard of the cockpit of a metropolitan train and a real seat. The geometry of the dashboard has been acquired through Reverse Engineering techniques from a real train dashboard. The user's immersion in the virtual environment during the simulation is guaranteed by the scene displayed on the Augmented Reality device, while, simultaneously, the stereoscopic projection on a screen above the dashboard makes available the experience even to users not directly involved, seeing the scene from the driver's point of view. The immersive Augmented Reality is realized through a Head-Mounted Display (HMD) by which the user, protagonist of the driving experience, sees the configuration of the virtual control devices (CAD geometries) overlapped with the physical dashboard in order to naturally interact into the immersive environment. The interaction between user and simulator happens through the NUI (Natural User Interfaces) based on markerless tracking of parts of the user's body.

Keywords: preventive safety, vitual simulator, railway design, vehicle driving, augmented reality, reverse engineering, train dashboard, head-mounted display, natural user interfaces, IDEAinVR.
\end{abstract}

\section{INTRODUCTION}

Use of Virtual Reality (VR) in design has an important potential in addition to the others already well known in literature [1], [2]: it makes possible the analysis and the improvement of requirements and safety features of the product, by the user or by people demanded to maintain it during its life cycle [3], before it is physically realized [4]. Today VR is used in all fields of product design, where it is a fundamental tool to improve the safety in environments not always suitable for the human presence, such as transportation systems [5], multi-physics [6], [7] or fusion field [8]-[10], especially coupled with other well-known design methodologies, such as systems engineering [11], [12].

The original and innovative aspect of the following activity is the use of techniques and methods of computer-aided design and virtual prototyping to improve the characteristics of "preventive safety" of a vehicle. The objective of preventive safety is to avoid dangerous situations, unlike the well-known objectives of passive safety, aimed at minimizing the consequences of an accident on the persons involved, and of active safety, whose purpose is to avoid the accident in dangerous situations. In particular, it is necessary to highlight the differences between the concepts of active safety, passive safety and preventive safety, with reference to the transportation field. In the first case, the ultimate goal of the design is the identification of systems and devices that intervene in order to prevent an adverse event, while, instead, passive safety provides for the adoption of devices that reduce the damage deriving from it (taking into account the interaction of the vehicle with the environment and 
its behaviour during a collision). Preventive safety, on the other hand, is implemented providing the vehicle with systems that prevent the occurrence of risk conditions that could lead to a possible accident. In the railway sector, the achievement of high travel speeds and the dramatic consequences associated with accidents entail greater difficulties in the intervention of active and passive safety devices, so it is evident how important is the attention paid to the development and improvement of preventive safety systems, increasingly efficient, particularly on the driver's cab and passenger compartments [13]. The technological objectives and methodologies developed have been validated through the design and the implementation of an experimental simulator at the IDEAinVR (Interactive Design and Ergonomics Applications in Virtual Reality) laboratory of the Department of Industrial Engineering (DII) (www.ideas.unina.it) of the University of Naples Federico II, which consists of a physical/virtual prototype of a driver's cab of a railway vehicle and a augmented reality environment for the simulation of driving operations.

In particular, the study is aimed at improving the perception of safety signals by a sample of users submitted to test session [14], [15]. After defining the test cases for the verification and validation of the functionalities present on the driver's diagnostic terminal, the possible simulation scenarios were defined, i.e. different proposals for setting up the driver's cab with relative arrangement of the command and signalling devices, to compare different concepts and select the best in terms of perception of the danger situation, reaction to an event and cognitive response in different situations of driving: Normal Road, Degraded March and Emergency Driving. The test session can provide different scenarios of metropolitan sections, with relative traction/braking curves, which must be travelled by the user in compliance with the prescribed driving methods, the signals and the events that occur in real time.

\section{METHODOLOGICAL APPROACH}

The methodology developed to carry out the analysis under consideration consisted in the realization of an Interactive Immersive Virtual Augmented Reality session in which the user lives the experience of a train driver, interacting with the train control panel and all its command and signal devices. In particular, the user is proposed and then experimented with different dashboards, different command layouts and different signalling and safety devices; according to the judgment that the user gives the different dashboard concepts, statistical analysis can be performed to understand the variation in the user's perception of safety as the different combinations of dashboards and devices vary, depending on its usability [16], [17].

\subsection{Immersive virtual reality}

Virtual reality, by its very definition, simulates actual reality. The progress of information technology allows to navigate in photorealistic worlds in real time [18], interacting with the objects present in them. The immersive virtual reality (an environment built around the user) according to the current technological level and according to the possible predictions for the near future, can be used by the mass thanks to some devices (partially already used): viewer, earphones, gloves and/or suit. The viewer can consist of a helmet or simple glasses in which the screens close to the eyes cancel the real world from the user's view, thus giving a feeling of immersive vision. The viewer can also contain systems for the detection of movements (accelerometer, gyroscope and magnetometer), so that moving the head will get the same action in the virtual environment. Gloves replace mouse, keyboard, joystick, trackball and other manual input systems. They can be used for movements, to give commands, to type on virtual keyboards, etc. The cybertuta is a suit that wraps the body and at the logical level extends to the whole body the features already described for the gloves. It can have multiple 
uses: it can simulate the touch by flexing on itself thanks to the elastic fabric, it can realize a three-dimensional tracing of the user's body and transfer it to the virtual environment (Fig. 1).

\subsection{Augmented Reality}

The term Augmented Reality means the overlapping of virtual elements, generated by the computer, to the perception of the real world, viewed through a camera or through special glasses. In general, Augmented Reality (AR) is the representation of an altered reality in which artificial, virtual information are overlapped on the normal reality perceived through the senses [19] (Fig. 2).

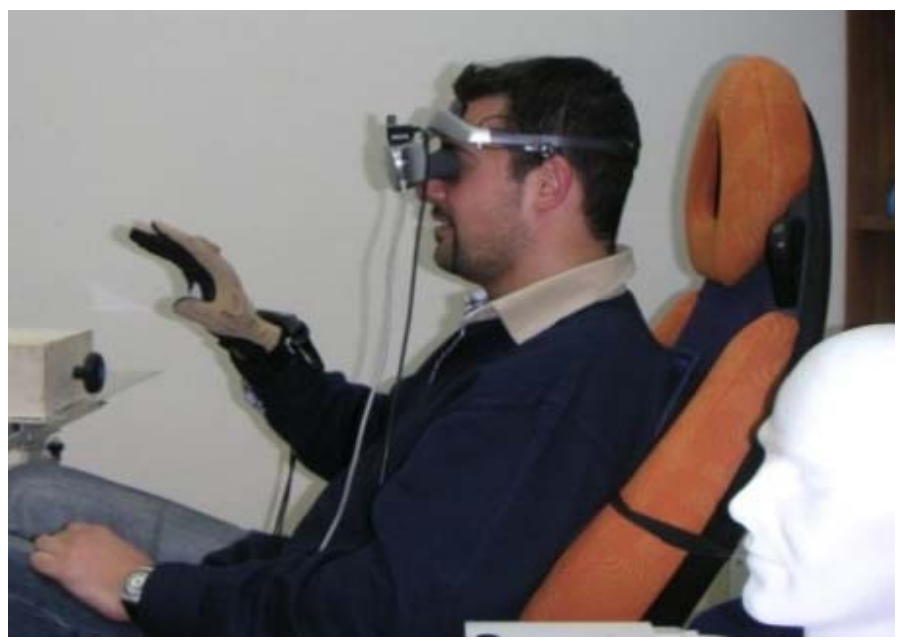

Figure 1: Devices suitable for the realization of an Immersive Virtual Reality.

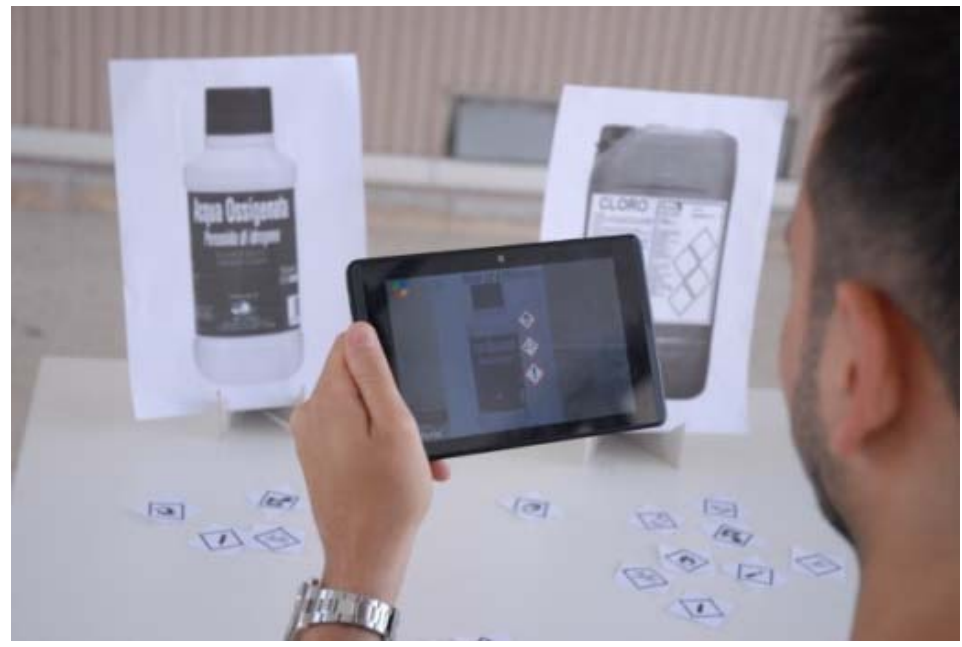

Figure 2: Example of Augmented Virtual Reality made usable by a tablet. 


\subsection{Interactive Virtual Reality}

Through the use of the latest generation of Motion Capture systems, it is possible to interact with Virtual Reality and not only enjoy it passively. In particular, there are devices that, through the use of RGB and/or infrared cameras, manage to "capture" the movement of a part of the body (for example the hands) or the entire body of a user and then reprocess this movement.

This technology can have essentially two purposes:

1. to issue commands to the software system using gesture recognition algorithms;

2. to move a digital manikin in the virtual world through the movements of the body.

This new paradigm of interaction with the digital world projects the user into the virtual world not only as a spectator of it, but as a real protagonist, giving him the opportunity to move and interact with the virtual scene with the realism and immersiveness of a task performed in the real world [20].

\section{HARDWARE ARCHITECTURE OF SIMULATOR}

To implement this methodology a simulator which provides the perfect integration of different technologies was developed. In particular, the simulator consists of a simulacrum of a dashboard of a metropolitan train cab and a real driver's seat, to support the simulation of driving tasks. The immersion of the user in the virtual environment during the simulation is guaranteed by the visualization of the scene in Immersive Augmented Reality, while, at the same time, the stereoscopic projection on a screen placed above the dashboard makes the experience usable even by users not directly involved, which see the scene taken from the point of view of the driver. The Immersive Augmented Reality is realized through a HeadMounted Display (HMD) through which the user, protagonist of the experience of the driver, sees perfectly overlaid the configuration of the virtual command devices (CAD geometries) with the geometry of the physical dashboard in order to interact naturally in the immersive environment. The geometry of the dashboard was acquired through synthetic Reverse Engineering techniques from a real one [21], [22]. User interaction with the simulator takes place through the NUI (Natural User Interfaces) based on the markerless tracing of parts of the user's body.

\subsection{Train dashboard}

The simulator presents a real command dashboard of a SIRIO tram. In order to have a perfect correspondence between the real component and the digital component, the real dashboard was acquired by laser scanning and reconstructed using the typical Reverse Engineering procedure (Fig. 3), chosen among different techniques [23]-[25].

\subsection{Kinect}

The Kinect sensor is equipped with an RGB camera, a dual infrared depth sensor consisting of an infrared laser scanner and a camera that is sensitive to the same band. The Kinect also features an array of microphones used by the system to calibrate the environment in which it is located, by analysing the reflection of sound on walls and furniture. In this way the background noises and ambient sounds are eliminated and voice commands can be correctly recognized (Fig. 4) [26]. 


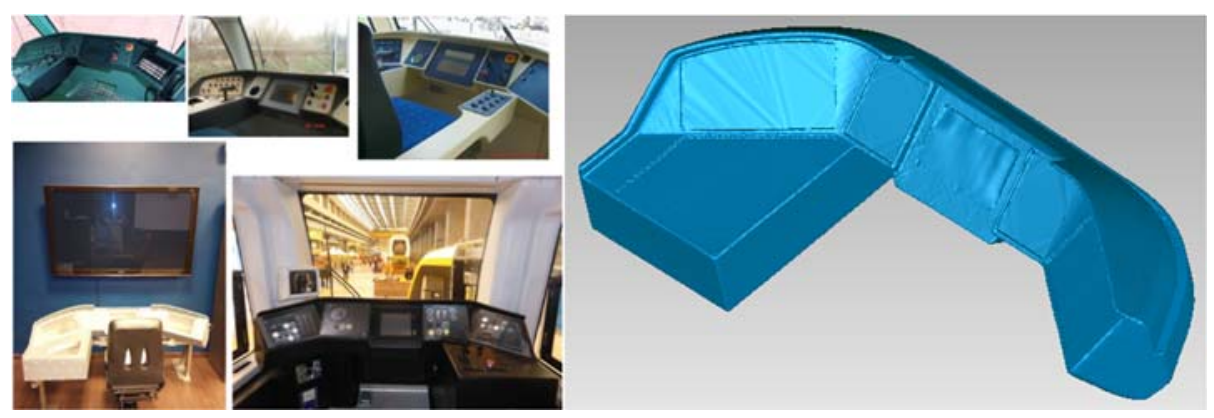

Figure 3: Driver dashboard of SIRIO Tram, reconstructed by RE techniques.
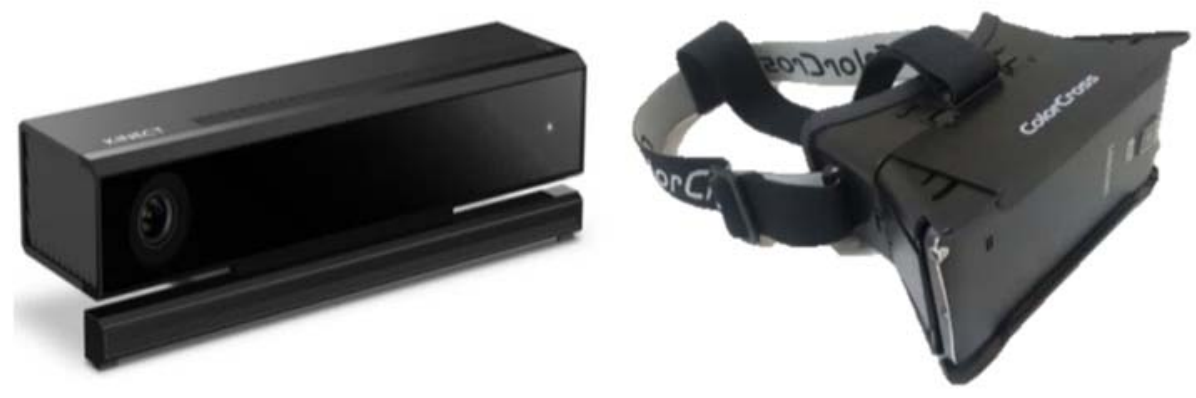

Figure 4: Kinect v2 Sensor and 3D Viewer.

In the simulator this sensor is used to track the movements of the user who simulates the driver's operations and thanks to Kinect the user's interaction with the Virtual Reality in which he is immersed is made possible.

\subsection{Head-Mounted Display}

The 3D viewer consists of a smartphone housed inside a wearable device, to realize a HeadMounted Display. This device allows to turn a smartphone into a Virtual Reality viewer. The viewer makes possible to experiment in an absolutely engaging and exciting way, in total visual immersion, the experience of Virtual Reality (Fig. 4).

The simulator has been equipped with a seat from a real train. The seat together with the dashboard makes the immersion offered by the railway simulator more realistic (Fig. 5).

\section{SOFTWARE ARCHITECTURE OF SIMULATOR}

The simulator was divided into two instances, both realized in the Unity environment [27][29]. The Server instance is a Windows application and has the task of managing most of the application logic and controls the screen for the public; the Client instance is an Android application and has the task of managing the HMD (Head-User Mounted Display). The Server instance has been connected to a Kinect For Windows V2 in order to collect information about the driver, the communication with the sensor was made with the official 


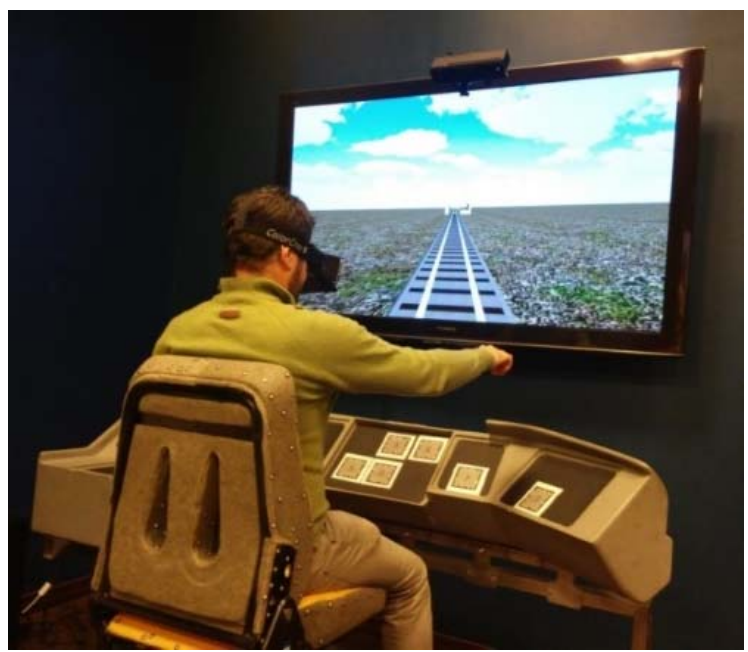

Figure 5: Virtual simulator of the cockpit of the railway vehicle in operation.

Microsoft driver package, in this way it is possible to directly read the position of the various parts of the body, in a three-dimensional Cartesian reference system where the Kinect in the $0,0,0$ position. Knowing the position of the user's head compared to the dashboard, with simple geometric calculations it is possible to identify the position of his limbs in relation to the dashboard, and so to identify the position of his hand in relation to virtual controls, such as buttons and levers. The Server instance at start up loads configuration features from the route to be travelled, the number of stations, the distance between stations, and the type of landscape between one station and another selected from Trees/Gallery/City; then, it dynamically generates the first 2000 meters of this route, every 1000 meters travelled the route is updated in order to present the user always with a value between 1000 and 2000 meters of distance. The position of the train is regulated by an "ideal" engine, i.e. not realistically simulated, controlled by a series of virtual objects, in particular an acceleration lever and a hydraulic braking button.

The Server instance also collects information both about the train and the whole journey (not only the currently displayed 1000-2000 meters) and through these data calculates the distance of the train from the following stations, the maximum speed that the train can have to stop at the next station using only the electric brake, and the phase of the stop/restart cycle in which the train is located. Then it uses this information to check the traffic lights of the stations and the indicators on the dashboard: speedometer, planner, speed indicator, brake lights, pantograph lights (Fig. 6).

The Client instance is connected to the server through a wireless connection managed by Unity, when the client instance is connected, the configuration file of the route is downloaded from the server and an identical one is built, then it continues to communicate with the server to maintain updated the status of the journey, the position of the train, the values indicated by the speedometer and planner, the status of the lights on the dashboard and the position of the user's hands as detected by Kinect. What's more, the Qualcomm Vuforia augmented reality engine and the HMD Google Cardboard system have been integrated into the client. Through Vuforia the application can recognize a series of markers, which use both to recognize with great precision the position and orientation of the head in relation to the 
dashboard, and the position, variable of the virtual instruments. Since each instrument is linked to an independent marker, moving the respective marker it is possible to move the instruments within the virtual scenario, creating the different configurations (Fig. 7).

The information on the position and orientation of the instruments, together with the information on the user, is constantly transmitted to the server, which uses them to establish the position of the user and all the instruments within the scenario in order to detect the interactions through the Kinect.

By integrating Vuforia with Google Cardboard, it is possible to realize a subjective stereoscopic vision of virtual controls in augmented reality, and it is also possible to continue to follow the rotation of the head even if the central marker linked to the dashboard is no longer framed.

\section{SCENARIOS AND TEST SESSIONS}

The possible simulation scenarios have been defined: that is, different for settings up the cockpit with the relative arrangement of the command and signalling devices, to compare different concepts and select the optimum in terms of perception of the danger situation, reaction to an event and cognitive response in different situations of driving the railway vehicle: Normal Road, Degraded March and Emergency Driving.
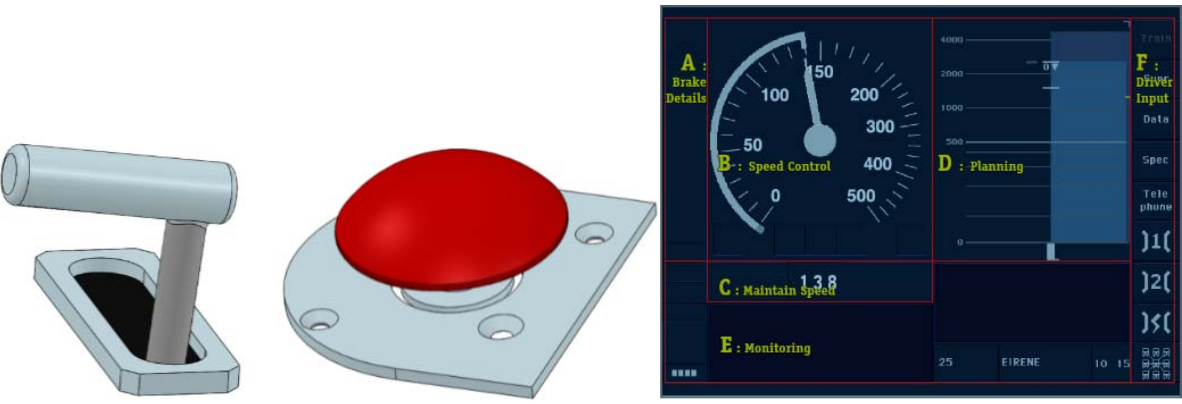

Figure 6: CAD models of traction lever and emergency braking button and train control interface divided into functional areas.
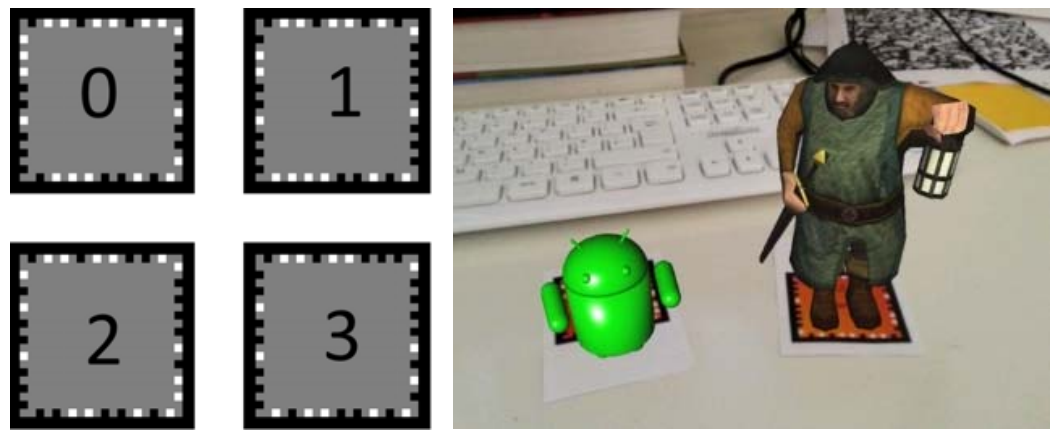

Figure 7: Augmented Reality realized through Vuforia marker recognition system. 


\subsection{Test sessions}

The test session can provide different scenarios of metropolitan sections, with relative traction/braking curves, which must be travelled by the user in compliance with the prescribed driving methods, the signals and the events that occur in real time [30]-[32], (Fig. 8).

\subsection{Speedometer status description}

This paragraph describes, as an example, the control/command device of the vehicle speedometer, inserted in the touchscreen positioned in the central part of the dashboard.

The possible states of the speedometer are 4 and depend on the Current Speed and the Speed Limits and of Intervention:

- $\quad$ current speed lower than the allowed speed limit (GREEN STATE);

- $\quad$ current speed equal to the permitted speed limit (GRAY STATE);

- current speed higher than the allowed speed limit but lower than the intervention speed (YELLOW STATE);

- $\quad$ current speed higher than the intervention speed (RED STATUS).

It is important to underline that the Current Speed is linked to the real movement of the (virtual) Gear Lever and that the Speed Limits and of Intervention are not fixed but are function of the mapping of the railway line and, therefore, updated according to the real position of the train on the line (GPS). Depending on the state in which they are located, there will be indicators indicating the driver's request for intervention, to be followed by an operation by the driver and consequent change of state of the vehicle or possible continuation of the previous state (if the operation performed by the driver was not that required/). A good vision and reading (interpretation) of the vehicle control and signalling devices are a key element of guarantee for a safe rail vehicle driving (Fig. 9).

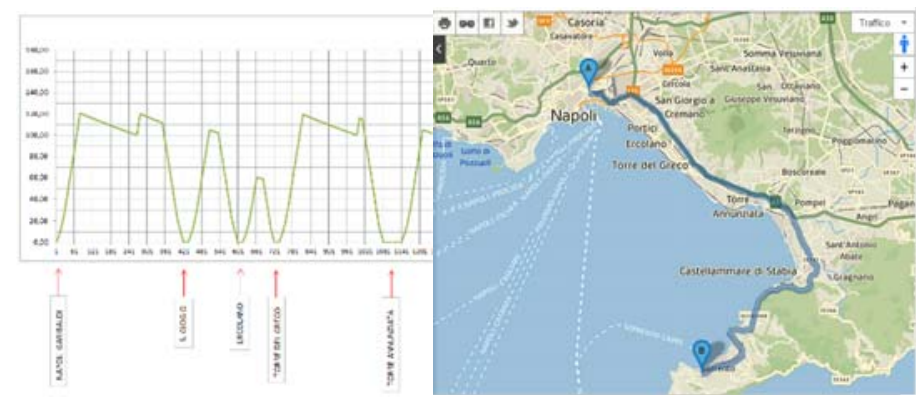

Figure 8: Example section of traction-braking curve implemented and corresponding road.

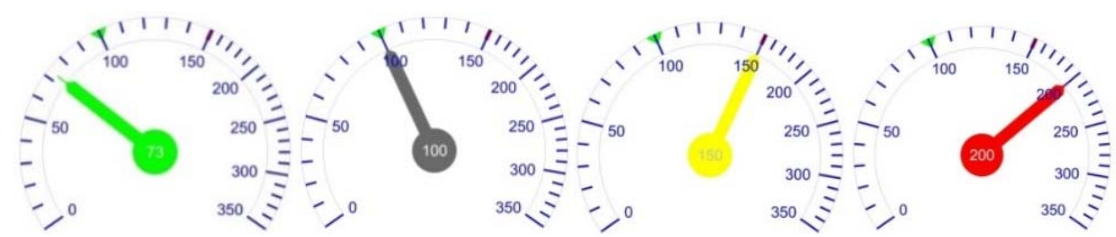

Figure 9: Different indicators according to the actual train speed during the simulation. 


\subsection{Features}

The main features of the designed simulator that intervene during the test sessions are described:

1. an algorithm for simulating the speed limits allowed and intervening, as if the vehicle were on a real operating line. These limits were simulated by implementing a traction-braking curve in the simulator;

2. a driver lever to increase and decrease the vehicle speed;

3. a script that reads the difference between vehicle speed and limits and updates in real time the colour of the indicators proposed for signalling to the driver of the change in status of the speedometer;

4. a button for emergency braking;

5. different types of dashboards:

6. open dashboard;

7. dashboard with side corridor;

8. dashboard designed for double drivers;

9. all the control devices are independent, so that they can be repositioned (even during a simulator test session) and active, so to be able to move the operating lever, press the emergency stop button, move the joystick of the dead man, etc.;

10. traffic lights, ground buoys and other signs on the route (quay with traffic lights).

The user who tests the simulator can simulate the activity of driver, try different tracks and perform demonstration sessions for statistical purposes in which he must (Fig. 10):

1. accelerate by moving the lever and observe the speed increase through the speedometer;

2. adjust the acceleration of the vehicle according to the signals on the speedometer;

3. perform an emergency stop following:
a. a warning signal on the dashboard;
b. an audio message that simulates a call from the ground;
c. a person on the tracks;

4. move "dead man" joystick;

5. operate pantograph, lowering or raising it according to the messages on the dashboard;

6. stop at one or more docks;

7. monitor various and possible failure and warning lights that can light up on the dashboard.

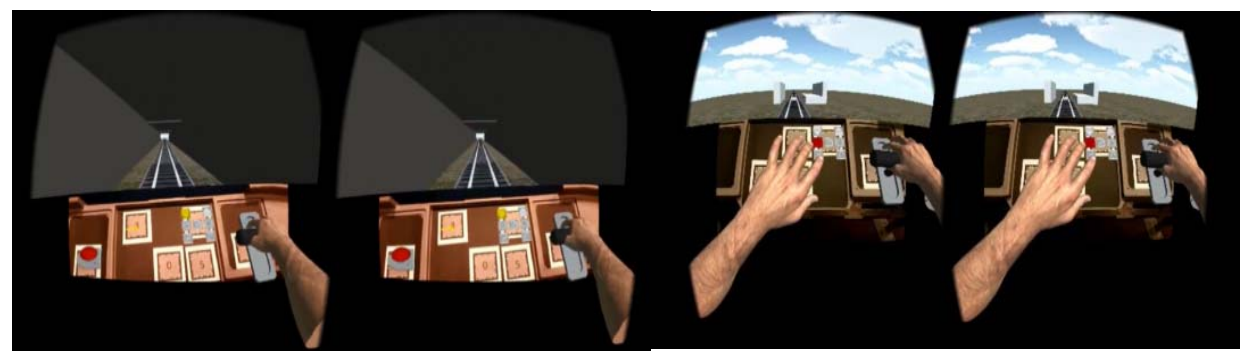

Figure 10: Test session of the simulator from the point of view of the driver. 

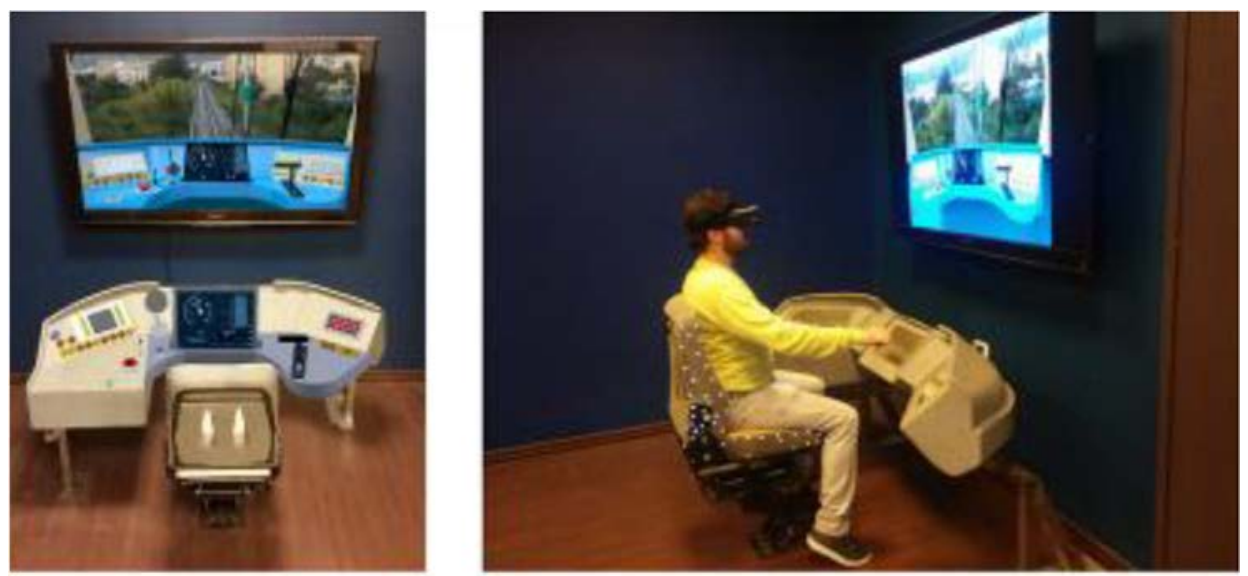

Figure 11: Final configuration of the simulator.

\section{CONCLUSIONS}

The purpose of this document is to describe the design, development and construction of a virtual simulator of the driving cab of a railway vehicle and the possible scenarios simulated with it, as well as the different proposals for the setting of the cockpit with the relative configuration of the control devices (Fig. 11).

After the test sessions, users were asked to fill in questionnaires related to their experience in Virtual Augmented Interactive Reality in terms of realism and usability [33]. The first feedback returned confirmed that the tool designed can certainly help evaluation to compare different concepts and select the optimal concept in terms of perception of the danger situation, reaction to an event and cognitive response in different situations of driving the railway vehicle. Statistical studies will be performed on the collected data in future works.

The aim is to make the driver concentrate as much as possible and to reduce the errors due to fatigue, allowing a customization of the instrumentation according to the actions that everyone acts most during the control. It will possible, by means deepening, development and engineering of final solutions to be launched on the market, to ensure the devices capable of performing the operations, after having been virtually tested in the simulator.

\section{ACKNOWLEDGEMENT}

The authors wish to thank Protom Group S.p.A. and Project "VERO - Virtual Engineering for railway and automotive" Campus for Industrial Research and Experimental Development issued by the Campania Region with Executive Decree n. 327, 11/8/2009, for technical support.

\section{REFERENCES}

[1] Di Gironimo, G. \& Lanzotti, A., Designing in VR. International Journal on Interactive Design and Manufacturing, 3(2), pp. 51-53, 2009.

[2] Renno, F. \& Papa, S., Direct modeling approach to improve virtual prototyping and FEM analyses of bicycle frames. Engineering Letters, 23(4), pp. 333-341, 2015.

[3] Di Gironimo, G., Mozzillo, R. \& Tarallo, A., From virtual reality to web-based multimedia maintenance manuals. International Journal on Interactive Design and Manufacturing (IJIDeM), 7(3), pp. 183-190, 2013. 
[4] Caputo, F., Di Gironimo, G. \& Papa, S.A., Virtual reality system for ergonomics and usability validation of equipment controls. Anales de Ingegnería Gráfica, 18, pp. 4764, 2006.

[5] Di Gironimo, G., Papa, S. \& Tarallo, A., Design review of train concepts in virtual reality. Proceedings of Eurographics Italian Chapter Conference, Trento, Italy, 2007.

[6] Papa, S., Patalano, S., Lanzotti, A., Gerbino, S. \& Choley, J.Y., Towards the integration of thermal physics and geometrical constraints for a 3D-multiphysical sketcher. Systems Engineering (ISSE), 2015 IEEE International Symposium on, pp. 248-252, IEEE, 2015.

[7] Lucca, F. et al., Preliminary electromagnetic, thermal and mechanical design for first wall and vacuum vessel of FAST. Fusion Engineering and Design, 98-99, pp. 1538$1542,2015$.

[8] Di Gironimo, G., Marzullo, D., Mozzillo, R., Tarallo, A. \& Villone, F., The DTT device: First wall, vessel and cryostat structures. Fusion Engineering and Design, 122, pp. 333-340, 2017.

[9] Labate, C., Di Gironimo, G. \& Renno, F., Plasma facing components: a conceptual design strategy for the first wall in FAST tokamak. Nuclear Fusion, 55(113013), 2015. DOI:10.1088/0029-5515/55/11/113013.

[10] Perri, G.M., Bräunig, M., Di Gironimo, G., Putz, M., Tarallo, A. \& Wittstock, V., Numerical modelling and analysis of the influence of an air-cooling system on a milling machine in virtual environment. The International Journal of Advanced Manufacturing Technology, 86(5-8), pp. 1853-1864, 2016.

[11] Marzullo, D., Bachmann, C., Coccorese, D., Di Gironimo, G., Mazzone, G. \& You, J.H., Systems engineering approach for pre-conceptual design of DEMO divertor cassette. Fusion Engineering and Design, 124, pp. 649-654, 2017.

[12] Meszaros, B., Shannon, M., Marzullo, D., Woodley, C., Rowe, S. \& Di Gironimo, G., Configuration management of the EU DEMO conceptual design data. Fusion Engineering and Design, 109, pp. 1619-1623, 2016.

[13] Di Gironimo, G. et al., knowledge-based engineering approach for supporting railway manufacturers from the tender notice to the designing phase. Computers in Railways XIV: Railway Engineering Design and Optimization, 135, p. 125, 2014.

[14] Ference, J.J., Szabo, S. \& Najm, W.G., Objective Test Scenarios for Integrated Vehicle-Based Safety Systems US 07-0183.

[15] Lanzotti, A., Di Gironimo, G., Matrone, G., Patalano, S. \& Renno, F., Virtual concepts and experiments to improve quality of train interiors. International Journal on Interactive Design and Manufacturing (IJIDeM), 3(2), pp. 65-79, 2009.

[16] Di Gironimo, G., Matrone, G., Tarallo, A., Trotta, M. \& Lanzotti, A., A virtual reality approach for usability assessment: case study on a wheelchair-mounted robot manipulator. Engineering with Computers, 29(3), pp. 359-373, 2013.

[17] Patalano, S., Lanzotti, A., Del Giudice, D.M., Vitolo, F. \& Gerbino, S., On the usability assessment of the graphical user interface related to a digital pattern software tool. International Journal on Interactive Design and Manufacturing, 11(3), pp. 457-469, 2017.

[18] Di Gironimo, G. \& Papa, S., Use of shader technology for realistic presentation of train prototypes in virtual reality. Eurographics Italian Chapter Conference, pp. 105-109, 2006.

[19] Lanzotti, A. et al., On the usability of augmented reality devices for interactive risk assessment. International Journal of Safety and Safety Engineering, 8(1), pp. 132-138, 2018. 
[20] Pelliccia, L. et al., Task-based motion control of digital humans for industrial applications. Procedia CIRP, 62, pp. 535-540, 2017.

[21] Gerbino, S., Del Giudice, D.M., Staiano, G., Lanzotti, A. \& Martorelli, M., On the influence of scanning factors on the laser scanner-based 3D inspection process. The International Journal of Advanced Manufacturing Technology, 84(9-12), pp. 17871799, 2016.

[22] Martorelli, M., Ausiello, P. \& Morrone, R., A new method to assess the accuracy of a Cone Beam Computed Tomography scanner by using a non-contact reverse engineering technique. Journal of Dentistry, 42(4), pp. 460-465, 2014.

[23] Grazioso, S., Selvaggio, M. \& Di Gironimo, G., Design and development of a novel body scanning system for healthcare applications. International Journal on Interactive Design and Manufacturing (IJIDeM), pp. 1-10, 2017.

[24] Calì, M., Speranza, D. \& Martorelli, M., Dynamic spinnaker performance through digital photogrammetry, numerical analysis and experimental tests. Lecture Notes in Mechanical Engineering, pp. 585-595, 2017.

[25] Martorelli, M., Pensa, C. \& Speranza, D., Digital photogrammetry for documentation of maritime heritage. Journal of Maritime Archaeology, 9(1), pp. 81-93, Springer, 2014. ISSN: 1557-2285.

[26] Pillai, I., Introduzione ai motion controllers ed al sensore Kinect POR Sardegna FESR, 2007/2013.

[27] https://unity3d.com/community.

[28] https://social.msdn.microsoft.com/forums/en-us/home?forum=kinectv2sdk.

[29] http://www.developerfusion.com/t/kinect/forum.

[30] UIC 651 (Layout of driver's cabs in locomotives).

[31] CEI EN 50155 - Applicazioni ferroviarie. Equipaggiamenti elettronici utilizzati sul materiale rotabile.

[32] EN 50125, Condizioni ambientali per equipaggiamenti a bordo del rotabile.

[33] Laugwitz, B., Held, T. \& Schrepp, M., Construction and evaluation of a user experience questionnaire. Symposium of the Austrian HCI and Usability Engineering Group, Springer: Berlin, Heidelberg, pp. 63-76. 2008. 